\title{
AVALIAÇÃO DE TÉCNICAS DE CLASSIFICAÇÃO DIGITAL DE IMAGENS LANDSAT EM DIFERENTES PADRÕES DE COBERTURA DA TERRA EM RONDÔNIA ${ }^{1}$
}

\author{
Allan Yu Iwama de Mello², Diogenes Salas Alves ${ }^{3}$, Claudia Albuquerque Linhares ${ }^{4}$ e \\ Fábio Bueno de Lima ${ }^{5}$
}

\begin{abstract}
RESUMO - Dados de sensoriamento remoto têm sido largamente utilizados para classificação da cobertura e uso da terra, em particular graças à aquisição periódica de imagens de satélite e à generalização dos sistemas de processamento digital de imagens, que oferecem uma variedade de algoritmos de classificação de imagens. Este trabalho teve por objetivo avaliar alguns dos métodos mais comuns de classificações supervisionadas e não supervisionadas para imagens do sensor TM do satélite Landsat-5, em três áreas com diferentes padrões de paisagem em Rondônia: (1) áreas de fazendas de "Médio porte", (2) assentamentos no padrão "Espinha de peixe" e (3) áreas de contato entre floresta e "Cerrado". A comparação com um mapa de referência baseado na estatística Kappa produziu indicadores de desempenho bons ou superiores (melhores resultados - K-médias: $\mathrm{k}=0,68 ; \mathrm{k}=0,77 ; \mathrm{k}=0,64$ e MaxVer: $\mathrm{k}=0,71 ; \mathrm{k}=0,89 ; \mathrm{k}=0,70$, respectivamente nas três áreas citadas), para os algoritmos utilizados. Os resultados indicaram que a escolha de um algoritmo deve considerar tanto a capacidade de discriminar várias assinaturas espectrais em diferentes padrões de paisagem quanto a relação custo/benefício decorrente das várias etapas do trabalho dos operadores que elaboram um mapa de cobertura e uso da terra. Este trabalho apontou a necessidade de esforço mais sistemático de avaliação prévia de várias opções de execução de um projeto específico antes de se iniciar o trabalho de elaboração de um mapa de cobertura e uso da terra.
\end{abstract}

Palavras-chave: Cobertura florestal, Classificação e Rondônia.

\section{CLASSIFICATION TECHNIQUES FOR LANDSAT TM IMAGERY UNDER DIFFERENT LANDSCAPE PATTERNS IN RONDÔNIA}

\begin{abstract}
Remotely sensed imagery has been widely used for land use/cover classification thanks to the periodic data acquisition and the widespread use of digital image processing systems offering a wide range of classification algorithms. The aim of this work was to evaluate some of the most commonly used supervised and unsupervised classification algorithms under different landscape patterns found in Rondônia, including (1) areas of mid-size farms, (2) "fish-bone" settlements and (3) a gradient of forest and Cerrado (Brazilian savannah). Comparison with a reference map based on the kappa statistics resulted in good to superior indicators (best results $-K$-means: $k=0.68 ; k=0.77 ; k=0.64$ and MaxVer: $k=0.71 ; k=0.89 ; k=0.70$ respectively for three areas mentioned). Results show that choosing a specific algorithm requires to take into account both its capacity to discriminate among various spectral signatures under different landscape patterns as well as a cost/benefit analysis considering the different steps performed by the operator performing a land cover/use map. it is suggested that a more systematic assessment of several options of implementation of a specific project is needed prior to beginning a land use/cover mapping job.
\end{abstract}

Keywords: Forest cover, Classification and Rondônia.

\footnotetext{
${ }^{1}$ Recebido em 26.03.2012 e aceito para publicação em 19.06.2012.

${ }^{2}$ Núcleo de Estudos e Pesquisas Ambientais, NEPAM/UNICAMP. E-mail: <allan.iwama@gmail.com>.

${ }^{3}$ Instituto Nacional de Pesquisas Espaciais, INPE, Brasil. E-mail: <dalves@ dpi.inpe.br>.

${ }^{4}$ Centro Nacional de Monitoramento e Alertas de Desastres Naturais, CEMADEN. E-mail: <claudia.linhares@ cemaden.gov.br>.

${ }^{5}$ Arvorar Soluções Florestais LTDA, ARVORAR, Brasil. E-mail: <fabio@ arvorar.com>.
} 


\section{INTRODUÇÃO}

Os estudos da cobertura florestal ou da cobertura e do uso da terra, sua dinâmica e seus impactos cresceram com os trabalhos sobre as mudanças ambientais nas últimas décadas (LAMBIN et al., 2003; MORAN e OSTROM, 2009). Os impactos das mudanças de cobertura e uso da terra estão associados, principalmente, ao comprometimento da biodiversidade, a mudanças no ciclo hidrológico, à degradação do solo e à capacidade dos sistemas biológicos em resistir às atividades humanas (RUDEL et al., 2005), mas também às dimensões sociais do problema (ALVES, 2007a; MORAN e OSTROM, 2009; ARAÚJO; LENÁ, 2010).

No caso da Amazônia, os processos predominantes de mudança da cobertura e uso da terra estão associados à conversão da floresta em áreas de pecuária e atividades agrícolas (SCHMINK; WOOD, 1992; MACHADO, 1998; MERTENS et al., 2002; MARGULIS, 2003; KAIMOWITZ et al., 2004; ALVES, 2007b). Entretanto, as causas presentes do desmatamento na Amazônia são diversas, frequentemente inter-relacionadas (SOARES-FILHO et al., 2005), podem variar significativamente com o tempo (FAMINOW, 1998; ALVES, 2007b) e mostrar diferentes associações com diversos padrões de paisagem (ALVES, 2002; MELLO; ALVES, 2011). Em particular, as atividades agrícolas têm seguido diferentes processos, que incluem distintos tipos de uso e intensificação da terra - rotação, degradação e abandono de terras (HECHT et al., 1988; FAMINOW, 1998; ALVES, 2007b; COSTA, 2008; ALVES et al., 2009). Além desses processos, configuram também o avanço da extração madeireira (NEPSTAD et al., 1999; NEPSTAD et al., 2001; ASNER et al., 2005; ASNER et al., 2006). A diversidade de padrões de mudanças de cobertura e uso da terra resulta numa grande variedade de respostas espectrais e de distintas combinações de arranjo, forma e tamanho de manchas, que também resultam num razoável grau de complexidade dos trabalhos de classificação e produção de mapas de cobertura e uso da terra.

Para diferenciar diferentes padrões de cobertura e uso da terra na Amazônia e suas mudanças, dados de sensoriamento remoto têm sido largamente utilizados como fonte principal para diferentes métodos de mapeamento (LU et al., 2004; ALVES et al., 2009). Dados de sensores Multispectral Scanner (MSS) e Thematic Mapper (TM) Landsat têm sido utilizados com diferentes técnicas de classificação e processamento de imagens (ex. TARDIN et al., 1980; SHIMABUKURO et al., 1982; SANO et al., 1989; SKOLE; TUCKER, 1993; MORAN et al., 1994; ALVES e SKOLE, 1996; ALVES, 2002; ALVES et al., 2003; BATISTELLA et al., 2003; FIDALGO et al., 2003; PONZONI; REZENDE, 2004; BATISTELLA; MORAN, 2005; ALVES, 2007a; ALVES et al., 2009), auxiliando a delimitação e quantificação de áreas de diferentes usos e coberturas da terra e compreensão de sua dinâmica.

Para adequada avaliação das diferentes técnicas de classificação, quando possível, devem ser considerados os seguintes fatores: (a) rigoroso tratamento do processo de registro e ortorretificação de imagens e correções atmosféricas (STEININGER, 2000; LU et al., 2004; ROBIN et al., 2007); (b) seleção criteriosa de dados/sensores, dada uma específica aplicação - diferentes sensores possuem diferentes resoluções espacial, temporal e espectral que influenciam os resultados e análises (FOODY et al., 1997; LU et al., 2004; VERBURG et al., 2011); (c) a qualidade e quantidade dos conjuntos de dados de treinamento para classes de interesse a serem mapeadas (LU et al., 2003; LU et al., 2004; VERBURG et al., 2011); (d) as variações do sensor entre as passagens do satélite - que influenciam na variabilidade espectral de determinada classe de uso (LU et al., 2004; VERBURG et al., 2011); (e) conhecimento e experiência da dinâmica espacial e temporal de ocupação do território analisado (LU et al., 2004); (f) uso de escala de análise adequada (LU et al., 2004; VERBURG et al., 2011); e ( $g$ ) agregação/ integração de diferentes métodos de classificação (FOODY et al., 1997; LU et al., 2004; ALVES et al., 2009; VERBURG et al., 2011). A seleção do método de classificação de imagens, muitas vezes, depende da diferenciação geográfica da área de estudo ou de interesse, sendo necessário iniciá-la pelo levantamento de dados disponíveis e, sobretudo, pela adequação desses dados ao problema sob investigação na área de estudo. Além disso, tem sido cada vez mais importante considerar aspectos sobre os diferentes padrões de paisagem para avaliação de técnicas de classificação de imagens, tema ainda pouco encontrado nos trabalhos de mapeamento de cobertura e uso da terra.

O objetivo deste trabalho foi avaliar o desempenho de métodos de classificação de imagens do sensor TM do satélite Landsat-5 em função de diferentes padrões de paisagem, que estão associados a tipos de cobertura e uso da terra diferentes. Os métodos 
foram avaliados na região de Rondônia, onde o desenvolvimento da agricultura e da pecuária em lotes de tamanhos variados (entre 25 e 1.000 ha - INCRA, 1996) resultou na formação de um mosaico complexo de padrões de uso e cobertura da terra.

Para essa avaliação, foram testados seis dos métodos de classificação automática mais utilizados, sendo quatro de classificação supervisionada (Máxima Verossimilhança, Paralelepípedo, Mahalanobis e Distância Mínima) e dois de classificação não supervisionada (K-médias e Isodata), bem como realizadas operações de edição manual em três áreas de diferentes padrões de paisagem em Rondônia, incluindo assentamentos no padrão espinha de peixe, áreas de fazendas de médio porte e áreas de contato floresta-cerrado. As classificações foram testadas com dados do sensor Thematic Mapper do satélite Landsat-5. Este trabalho visou contribuir para melhor entendimento do uso de técnicas de classificação digital em áreas com diferentes padrões de cobertura e uso da terra.

\section{MATERIAIS E MÉTODOS}

Para a classificação foram utilizadas imagens do sensor Thematic Mapper (TM) - Landsat-5 de 1985, correspondentes às órbitas/ponto 231/67 e 230/68, no Estado de Rondônia. Essas imagens foram previamente georreferenciadas, utilizando-se coordenadas adquiridas em campo com um Differential Global Positioning System - DGPS (ALVES et al., 2003; LINHARES et al., 2007).

Foram feitos recortes de 800 colunas $\mathrm{x} 400$ linhas das cenas, nas bandas 3 (vermelho), 4 (infravermelho próximo) e 5 (infravermelho médio), sendo cada recorte relativo a um padrão de uso e cobertura da terra. $\mathrm{O}$ processamento das imagens foi realizado no aplicativo ENVI versão 4.3 .

\section{1. Área de Estudo}

A área de estudo está localizada no Estado de Rondônia e foi dividida em três subáreas com diferentes padrões de cobertura e uso da terra (Figura 1): (1) "Fazendas de médio porte" (denominadas "Médio porte" no restante do artigo), situada em parte do Município de Ariquemes, o qual apresenta grande área de floresta e parcelas maiores que 500 ha, onde predominam pecuária; (2) "Espinha de peixe", entre os Municípios de Ministro Andreazza e Cacoal, com lotes de algumas dezenas de hectares num arranjo regular no típico padrão em "espinha de peixe", de assentamentos rurais do INCRA, onde ocorrem áreas de culturas agrícolas e pecuária; e (3) Contato floresta/Cerrado (denominada "Cerrado" no restante do trabalho), no Município de Pimenta Bueno, na confluência dos rios Comemoração e Ji-Paraná, apresentando grande área de Cerrado entremeando as áreas desflorestadas. A utilização de imagens de um período intermediário da ocupação de Rondônia (1985) teve o intuito de testar o desempenho dos algoritmos em diferentes configurações da paisagem que não mostrassem, entretanto, combinações muito heterogêneas de usos da terra.

As classes finais utilizadas para classificação dos padrões de cobertura florestal foram (1) "Médio porte" - floresta, capoeira, solo exposto, afloramentos rochosos e indícios de extração madeireira; (2) "Espinha de peixe" - floresta, capoeira e solo exposto; e (3) "Cerrado" - floresta, capoeira, solo exposto e Cerrado. Para diferenciar as classes de cobertura e uso da terra, foram utilizados, em primeira instância, os critérios de que pastagens e áreas de solo exposto e pouca vegetação apresentam maiores reflectâncias do vermelho e do infravermelho médio, em comparação com as áreas de floresta e de capoeira (vegetação secundária) (PEREIRA; SETZER, 1993); e a classe capoeira , apesar de maior grau de confusão com a floresta, mostrou maiores valores no infravermelho próximo em áreas de fase inicial da vegetação secundária e apresentou diminuição gradual do infravermelho próximo quando a regeneração apresentava estágio mais avançado (STEININGER, 2000). A identificação da classe Cerrado se baseou em Silva et al. (2008).

\subsection{Métodos de classificações supervisionadas e não supervisionadas}

As classificações testadas foram: (i) Supervisionadas (MaxVer, Mahalanobis, Paralelepípedo e Distância Mínima) e (ii) Não supervisionadas (K-médias e Isodata). Nos métodos de classificação supervisionados, foram selecionadas amostras de treinamento para cada classe da legenda, através de análise visual das feições cobertura e uso da terra. Nos métodos não supervisionados, após vários testes foram definidos os seguintes números de classes e iterações: (a) K-médias: "Médio porte" - 9 classes e 10 iterações; "Espinha de peixe" - 5 classes e 6 iterações; "Cerrado" - 10 classes e 10 iterações; (b) Isodata:

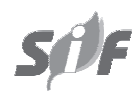

Revista Árvore, Viçosa-MG, v.36, n.3, p.537-547, 2012 


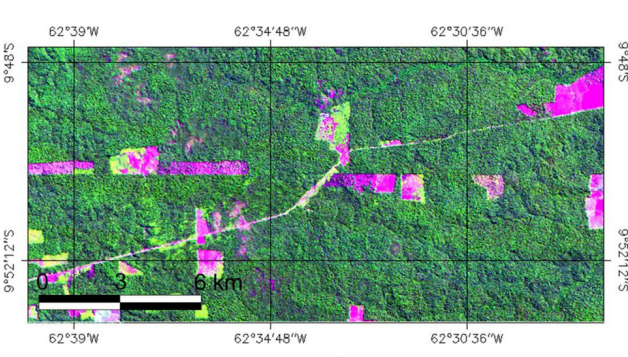

(1)

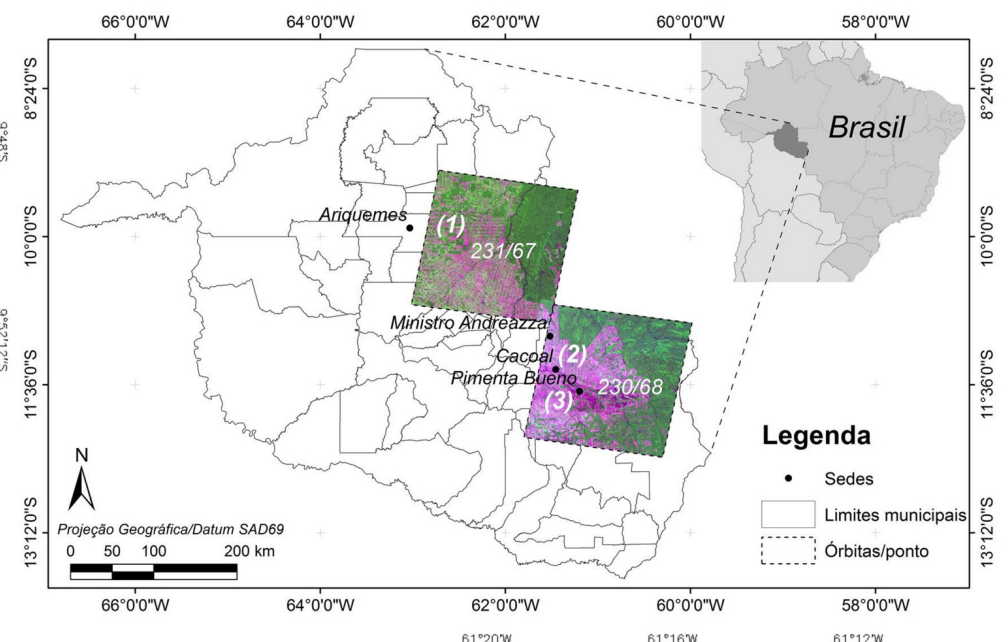

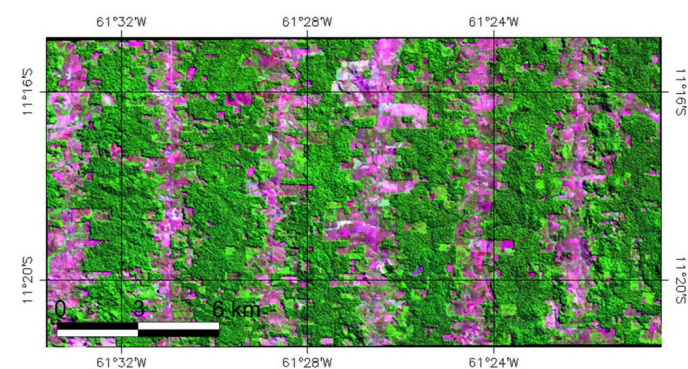

(2)

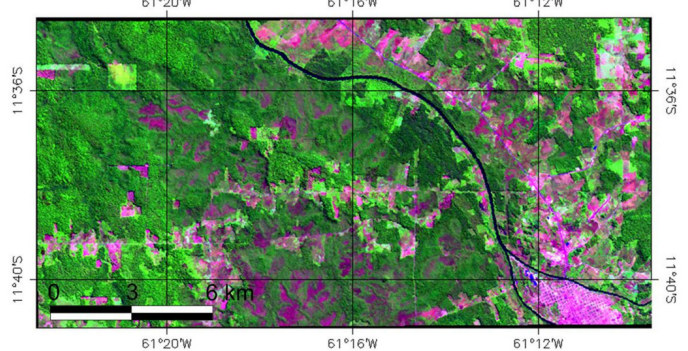

(3)

Figura 1 - Área de estudo: Estado de Rondônia e a localização das três sub-áreas: (1) "Médio porte", (2) "Espinha de peixe", (3) "Cerrado". Imagens TM/Landsat-5, composição colorida falsa cor.

Figure 1 -Study area: Rondônia state and the three sub-areas of study: (1) Mid-size farms, (2) "Fish-bone" settlements and (3) Forest/Cerrado (Brazilian savannah) gradient. Thematic Mapper (TM) Landsat-5 synthetic color imagery.

"Médio porte" - até 8 classes iniciais e 8 iterações; "Espinha de peixe" - até 5 classes iniciais e 6 iterações; e "Cerrado" - até 10 classes iniciais e 10 iterações. As classes obtidas nesse caso foram associadas às classes da Tabela 1, seguindo-se procedimentos de interpretação.

\subsection{Avaliação do desempenho pela estimativa do índice de concordância das classificações}

Para cada classificação de cobertura e uso da terra ("Médio porte", "Espinha de peixe" e "Cerrado"), foi feita uma amostragem estratificada aleatória de $15 \%$ dos pixels. Com as amostras selecionadas de cada padrão de paisagem, foram geradas matrizes de confusão.

Para estimar a concordância entre as classificações, foi aplicado o teste de significância entre dois coeficientes Kappa independentes, em determinado nível de significância. Congalton e Mead (1983) sugeriram a equação para esse teste.

$$
Z=\frac{\hat{\mathrm{K}}_{1}-\hat{\mathrm{K}}_{2}}{\sqrt{\hat{\sigma}_{\mathrm{k} 1}+\hat{\sigma}_{\mathrm{k} 2}}}
$$

em que:

$\mathrm{Z}=$ valor $\mathrm{Z}$ calculado;

$\mathrm{K}_{1}$ = estimativa do coeficiente Kappa para o classificador MaxVer;

$\mathrm{K}_{2}$ = estimativa do coeficiente Kappa para o classificador K-médias;

$\sigma_{\mathrm{k} 1}=$ estimativa da variância do coeficiente Kappa para o classificador MaxVer; e

$\sigma_{\mathrm{k} 2}=$ estimativa da variância do coeficiente Kappa para o classificador K-médias. 
A variância de Kappa foi estimada, segundo Bishop et al. (1975), utilizando-se a equação 2.

$\hat{\sigma}_{\mathrm{k}}=\frac{1}{\mathrm{n}}\left[\frac{\theta_{1}\left(1-\theta_{1}\right)}{\left(1-\theta_{2}\right)^{2}}+\frac{2\left(1-\theta_{1}\right)\left(2 \theta_{1} \theta_{2}-\theta_{3}\right)}{\left(1-\theta_{2}\right)^{3}}+\frac{\left(1-\theta_{1}\right)^{2}\left(\theta_{4}-4 \theta_{2}^{2}\right)}{\left(1-\theta_{2}\right)^{4}}\right]$ em que o valor $Z$ calculado para o teste foi comparado com o valor $Z$ tabelado $(Z$ tabelado $=1,96)$, a $5 \%$ de probabilidade, a fim de identificar se os dois classificadores de cada padrão apresentavam diferenças significativas ou não.

Para análise qualitativa dos valores de Kappa, os resultados foram comparados com base nos intervalos definidos em Landis e Koch (1977). Ao final foi feita a interpretação visual das três áreas, a fim de comparar o desempenho dessa técnica em relação aos métodos de classificação.

\section{RESULTADOS E DISCUSSÃO}

A avaliação do desempenho dos algoritmos de classificação automática e da interpretação visual e através do índice de concordância Kappa é apresentada na Tabela 1, e os resultados obtidos pelas classificações que mostraram os melhores índices Kappa são mostrados na Figura 2.

Na Tabela 1, os resultados da classificação podem ser considerados como bons, muito bons e excelentes (LANDIS; KOCH, 1977). Nos testes realizados, o método de classificação não supervisionado que apresentou maiores valores de Kappa nas três áreas foi o algoritmo K-médias (desempenho muito bom). Entre os métodos supervisionados, o melhor resultado foi obtido pelo classificador de máxima verossimilhança (MaxVer) (desempenho muito bom a excelente). A comparação entre os índices de concordância dos algoritmos MaxVer e K-Médias pelo teste $\mathrm{Z}$ mostrou diferenças significativas (5\% de significância). Ao mesmo tempo, os resultados da interpretação visual produziram os melhores índices de concordância em todas as áreas-teste.
De forma geral, esses resultados mostram, de um lado, diferenças de desempenho entre os vários algoritmos, mas de outro, desempenhos que podem ser classificados como superiores ou iguais a bom em todos os casos (LANDIS; KOCH, 1977), sugerindo a utilidade de analisar como a diversidade de respostas espectrais pode afetar o desempenho dos algoritmos. Isso foi feito para os classificadores que apresentaram os melhores desempenhos dos grupos supervisionado (MaxVer) e não supervisionado (K-Médias), através da análise das respectivas matrizes de confusão.

Em relação à área de "Médio porte" (Tabela 2a), os valores das diagonais das matrizes de confusão sugerem que as classes "capoeira", "solo exposto" e "afloramentos rochosos" foram mais bem mapeadas com o K-médias, enquanto a classe "floresta" foi com o MaxVer.

Independentemente do classificador, os maiores erros de omissão das classes "afloramentos rochosos" e "extração madeireira" ocorreram na classe "floresta". A resposta espectral da classe "extração" consiste em uma combinação de dois alvos: "floresta" e "solo exposto", em diferentes proporções, dependendo do grau de retirada de árvores e do número de clareiras abertas. O mapeamento dos "afloramentos rochosos", por apresentar padrão espectral diferente dos demais alvos, foi beneficiado pelo uso do K-médias, que foi capaz de distinguir essas áreas, enquanto o procedimento de aquisição de amostras para o Max Ver não o fez. No entanto, houve confusão com a classe "capoeira", provavelmente por causa dos diferentes estágios que a capoeira pode apresentar. A classe "capoeira" foi sistematicamente confundida com "floresta" e "extração" no MaxVer, pelo fato de o seu padrão espacial ser heterogêneo, apresentando manchas de solo entremeadas por manchas de vegetação, podendo ser confundida, em alguns pontos, com áreas de extração. Foi observado que a confusão com floresta ocorreu frequentemente nas bordas dos polígonos. De maneira geral, verificou-

Tabela 1 - Coeficiente de concordância Kappa para as classificações.

Table 1 - Kappa coefficient of agreement for the classifications.

\begin{tabular}{lccccccc}
\hline Áreas de estudo & \multicolumn{3}{c}{ Não supervisionado } & \multicolumn{2}{c}{ Supervisionado } & \multicolumn{2}{c}{ Interpretação visual } \\
\hline & Isodata & K-médiasMínima & Distância & Mahalanobis & Paralelepípedo & MaxVer \\
\hline Médio porte & 0,677 & 0,686 & 0,539 & 0,445 & 0,454 & 0,709 & 0,8115 \\
Espinha de peixe & 0,766 & 0,773 & 0,84 & 0,807 & 0,77 & 0,89 & 0,9254 \\
Cerrado & 0,553 & 0,642 & 0,653 & 0,629 & 0,693 & 0,704 & 0,7871 \\
\hline
\end{tabular}




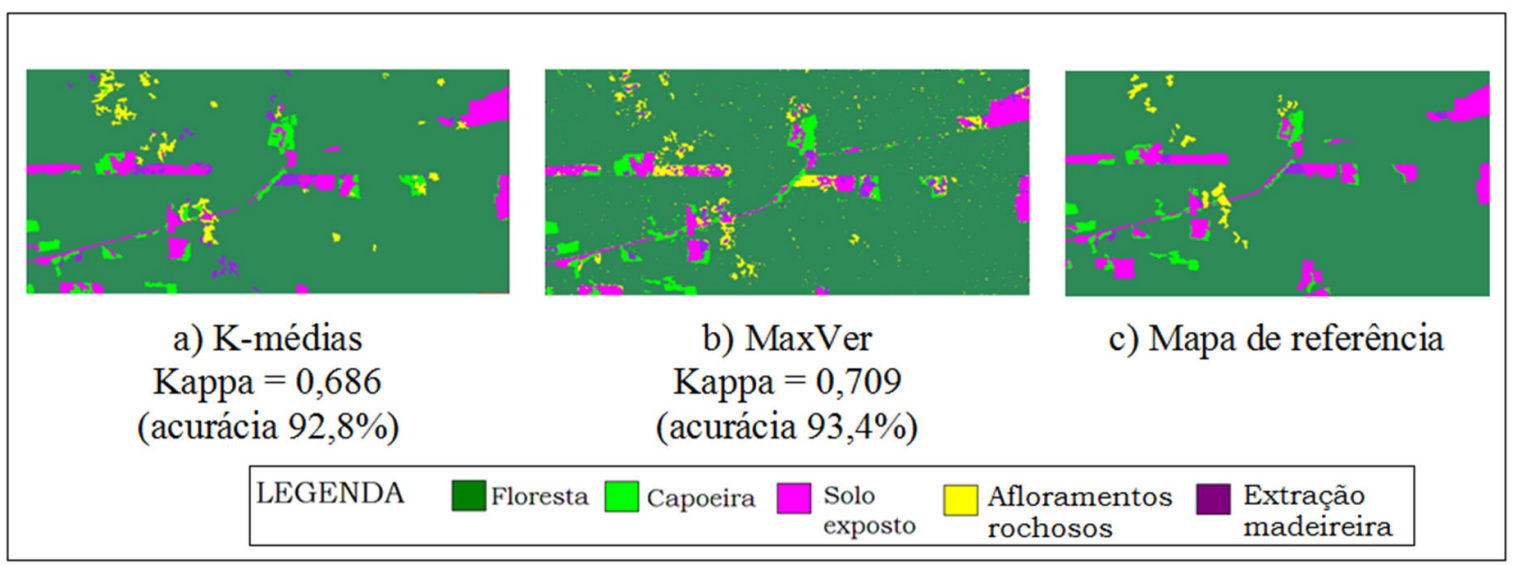

(1)

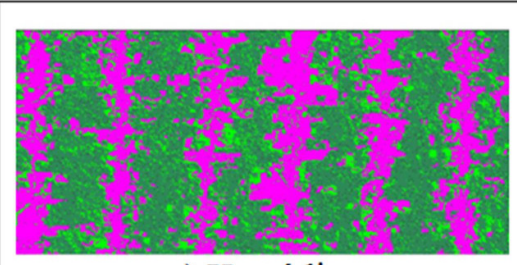

a) K-médias

$\mathrm{Kappa}=0,773$

(acurácia 75\%)

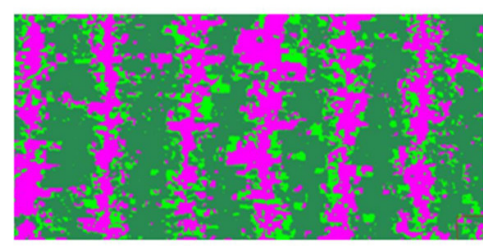

b) MaxVer

Kappa $=0,890$

(acurácia 88,4\%)

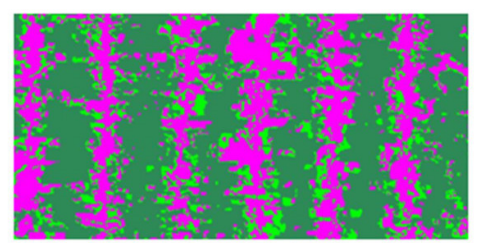

c) Mapa de referência

LEGENDA $\square$ Floresta $\square$ Capoeira $\square$ Solo exposto

(2)

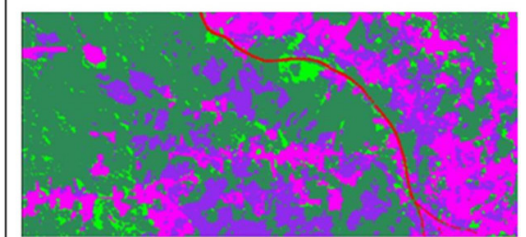

a) K-médias

Kappa $=0,642$

(acurácia 76\%)

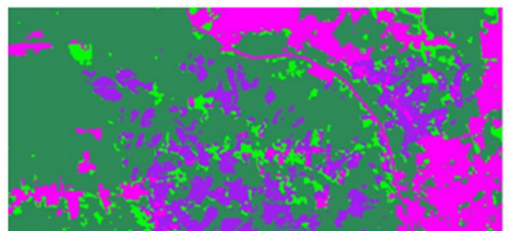

b) MaxVer

Kappa $=0,704$

(acurácia 80,5\%)

\section{LEGENDA $\square$ Floresta $\square$ Capoeira $\square$ Solo exposto $\square$ Cerrado}

(3)

Figura 2 - Resultado das classificações para os melhores resultados (1) "Médio porte", (2) "Espinha de peixe" e (3) "Cerrado".

Figure 2 -Best classification results: (1) Mid-size farms, (2) "Fish-bone" settlements and (3) Forest/savannah gradient (Cerrado). The classes in the figures Floresta, Capoeira, Solo exposto, and Cerrado, correspond, respectively, to Forest, Regrowth/Secondary vegetation, Bare soil, and Cerrado (Brazilian savannah).

Revista Árvore, Viçosa-MG, v.36, n.3, p.537-547, 2012 
Tabela 2 - Matriz de confusão (\%) para a área $(a)$ "Médio porte", $(b)$ "Espinha de peixe", $(c)$ "Cerrado".

Table 2 - Confusion matrix (\%): (a) Mid-size farms, (b) "Fish-bone" settlements and (c) Forest/Cerrado (Brazilian savannah) gradient.

\begin{tabular}{|c|c|c|c|c|c|c|c|c|}
\hline \multirow{2}{*}{ Padrão } & & \multirow{2}{*}{ Classificação } & \multicolumn{5}{|c|}{ Referência } & \multirow{2}{*}{ Total } \\
\hline & & & Floresta & Solo exposto & Afloramentos rochosos & Capoeira & Extração & \\
\hline \multirow{12}{*}{ 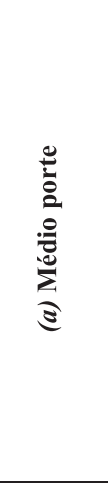 } & \multirow{6}{*}{ MaxVer } & Floresta & 97,3 & 6,1 & 40 & 25 & 50 & 88,8 \\
\hline & & Solo exposto & 1,6 & 75,8 & 0 & 0 & 0 & 6,4 \\
\hline & & Afloramentos rochosos & 0 & 9,1 & $\mathbf{0}$ & 0 & 0 & 0,6 \\
\hline & & Capoeira & 0,4 & 6,1 & 0 & 75 & 0 & 2,6 \\
\hline & & Extração & 0,7 & 3 & 60 & 0 & 50 & 1,6 \\
\hline & & Total & 100 & 100 & 100 & 100 & 100 & 100 \\
\hline & \multirow{6}{*}{ K-médias } & Floresta & 95,8 & 9,4 & 20 & 16,7 & 50 & 87,4 \\
\hline & & Solo exposto & 0,9 & 78,1 & 20 & 0 & 0 & 6 \\
\hline & & Afloramentos rochosos & 2 & 0 & 20 & 0 & 0 & 2 \\
\hline & & Capoeira & 0,2 & 9,4 & 40 & 83,3 & 0 & 3,2 \\
\hline & & Extração & 1,1 & 3,1 & 0 & 0 & 50 & 1,4 \\
\hline & & Total & 100 & 100 & 100 & 100 & 100 & 100 \\
\hline \multirow{2}{*}{ Padrão } & \multirow{2}{*}{\multicolumn{2}{|c|}{ Classificação }} & \multicolumn{5}{|c|}{ Referência } & Total \\
\hline & & & Floresta & \multicolumn{2}{|c|}{ Solo Exposto } & \multicolumn{2}{|c|}{ Capoeira } & \\
\hline \multirow{8}{*}{ 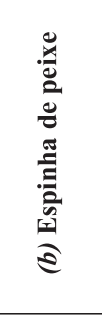 } & \multirow{4}{*}{ MaxVer } & Floresta & 96,2 & \multicolumn{2}{|r|}{$\begin{array}{c}0 \\
\mathbf{0 3}\end{array}$} & \multicolumn{2}{|c|}{14,1} & 53 \\
\hline & & Solo Exposto & 1,9 & \multicolumn{2}{|r|}{93} & \multicolumn{2}{|c|}{1,3} & 30,6 \\
\hline & & Capoeira & 1,9 & \multicolumn{2}{|r|}{7} & \multicolumn{2}{|c|}{84,6} & 16,4 \\
\hline & & Total & 100 & \multicolumn{2}{|r|}{100} & \multicolumn{2}{|c|}{100} & 100 \\
\hline & \multirow{4}{*}{ K-médias } & Floresta & 89,4 & \multicolumn{2}{|r|}{2,5} & \multicolumn{2}{|c|}{19,5} & 51 \\
\hline & & Solo Exposto & 0 & \multicolumn{2}{|r|}{95} & \multicolumn{2}{|c|}{22,1} & 33,6 \\
\hline & & Capoeira & 10,6 & & 2,5 & 58, & & 15,4 \\
\hline & & Total & 100 & & 100 & 100 & & 100 \\
\hline Padrão & & Classificacão & & & Referência & & & Total \\
\hline & & & Floresta & Capoeira & Solo exposto & Cerra & & \\
\hline & & Floresta & 95,4 & 21,1 & 5,1 & 21 & & 56,4 \\
\hline & MaxVer & Capoeira & 0,8 & 59,6 & 9,3 & 3,2 & & 9,8 \\
\hline & & Solo exposto & 1,9 & 14 & 67,8 & 8,1 & & 19,6 \\
\hline 웅 & & Cerrado & 1,9 & 5,3 & 17,8 & 67 & & 14,2 \\
\hline$\stackrel{\pi}{0}$ & & Total & 100 & 100 & 100 & 100 & & 100 \\
\hline 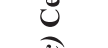 & & Floresta & 85,5 & 3,5 & 2,5 & 12 , & & 47,4 \\
\hline e & K-médias & Capoeira & 3,4 & 38,6 & 0 & 0 & & 6,2 \\
\hline & 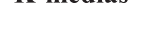 & Solo exposto & 0,8 & 54,4 & 71,4 & 4,8 & & 24,2 \\
\hline & & Cerrado & 10,3 & 3,5 & 26,1 & 82 & & 22,2 \\
\hline & & Total & 100 & 100 & 100 & 10( & & 100 \\
\hline
\end{tabular}

se que nas transições de uma classe para a outra, ou seja, nas bordas dos polígonos, houve pior desempenho dos classificadores, devido à mistura de respostas espectrais das duas classes adjacentes no pixel.

Nas áreas de "capoeira”, compostas por vegetação secundária em diferentes estágios, é comum a ocorrência de confusão com a classe "floresta", à medida que a estrutura avança em direção ao porte florestal. Dessa forma, os classificadores não supervisionados, agindo livremente e baseados apenas na diferenciação espectral dos alvos, podem tender a atribuir a classe floresta a vários pixels da classe "capoeira", gerando erros de omissão maiores do que no uso de classificadores supervisionados, quando são fornecidas amostras ao classificador. 
O “solo exposto", apesar do padrão espacial mais homogêneo que apresenta nessa área-teste, mostrou certo grau de confusão "capoeira" e "afloramentos rochosos" no MaxVer, e com "floresta" e "capoeira" no K-médias. Considerando que no MaxVer são fornecidas amostras das classes para o algoritmo e cada pixel é classificado de acordo com essa referência, é preciso ressaltar que tanto as áreas de extração quanto as de "afloramentos rochosos" apresentam proporção de solo exposto, sendo provável que haja confusão entre essas classes. Já o K-médias identifica automaticamente $\mathrm{N}$ classes na imagem (geralmente maior que o número de classes da legenda adotada), as quais o usuário pode agrupar a seu critério. Áreas de solo exposto que apresentaram sinais de vegetação de baixo porte provavelmente foram classificadas como um grupo à parte e reagrupadas com a classe "capoeira". A confusão com floresta ocorreu nas bordas, no contato com a floresta, como observado anteriormente.

A classe "floresta" possui resposta espectral bem definida, apresentando baixos erros de omissão, e em ambos os classificadores, a despeito das áreas de sombra, que costumam confundir o algoritmo.

Na área da "Espinha de peixe", o MaxVer apresentou desempenho superior ao de K-médias, com baixos erros de omissão/inclusão, ou seja, poucas confusões entre classes (Tabela 2b). Analisando os valores na diagonal, notaou-se que o K-médias distinguiu melhor a classe "solo exposto", enquanto as classes "floresta" e "capoeira" foram mais bem discriminadas pelo Max Ver. Os maiores erros, em ambos os classificadores, estiveram relacionados à confusão entre "capoeira" e as classes "floresta" e "solo exposto". Entre "capoeira" e "floresta", a explicação é a semelhança espectral entre ambas, já entre "capoeira" e "solo exposto" é o fato de algumas amostras de capoeira se encontrarem em estágios iniciais, com grandes proporções de solo exposto e algumas amostras de pastos apresentarem contribuição de resposta a gramíneas.

Na área de "Cerrado", os valores da diagonal indicaram que o MaxVer mostrou-se de melhor classificação para "floresta" e "capoeira", enquanto "solo exposto" e "cerrado" foram mais bem classificados com K-médias (Tabela 2c). O classificador K-médias apresentou maior erro de inclusão da classe "capoeira" em "solo exposto" (cerca de 54\%), possivelmente pelos motivos já expostos anteriormente. Outro erro de inclusão correspondeu a $26 \%$ das áreas de "solo exposto" classificadas como "Cerrado", devido à semelhança das respostas espectrais de ambos. No caso contrário, ou seja, "Cerrado" classificado erroneamente como "solo exposto", o erro observado foi menor, devido ao uso de uma máscara sobre o Cerrado. Por fim, entre $10 \%$ e $25 \%$ das áreas de "capoeira" e de "Cerrado" foram erroneamente classificadas como "floresta". A classe "floresta" novamente foi bem caracterizada com ambos os classificadores.

Diferenças no desempenho dos classificadores entre as áreas de estudo podem ser inferidas comparando-se as matrizes de confusão da Tabela 2 a-c. As áreas de "Cerrado" e "Médio porte" mostraram, de forma geral, os erros mais frequentes e elevados, enquanto na "Espinha de peixe" houve relativamente menos confusão e os erros de omissão e de inclusão foram mais baixos. As diferenças entre os classificadores dependem, entre outros fatores, da diversidade de respostas espectrais encontradas em cada área. Nessa ótica, é importante observar-se que a área de "Médio porte" mostra um padrão espacial relativamente simples e classes espectrais mais homogêneas, fatores que, em princípio, poderiam simplificar o mapeamento e propiciar melhores resultados. Entretanto, a área de "Cerrado" apresenta maior complexidade espacial e classes espectralmente ambíguas, fatores que dificultam o mapeamento. No entanto, apesar dessas disparidades, ambas as áreas apresentaram resultados inferiores quando comparadas com "Espinha de peixe", que teve os menores erros de omissão e de inclusão, apesar de os padrões espacial e espectral serem medianamente complexos.

Tomados em seu conjunto, os resultados desta avaliação indicaram que diferentes classificadores podem oferecer diferentes desempenhos e se, por um lado, todos os classificadores avaliados neste trabalho mostraram desempenhos classificados como "bons" ou superiores, por outro diversos outros fatores devem ser considerados pela pessoa ou equipe que executará um trabalho de classificação de forma a otimizar os custos do trabalho, conforme o algoritmo escolhido. Adiversidade de respostas espectrais da área de estudo deve estar associada ao número de classes desejado, porém é crucial identificar corretamente as possíveis fontes de confusão espectral e os custos de correção dos erros de classificação. Para isso, é importante, de um lado, que o trabalho de classificação considere as especificidades do funcionamento de cada algoritmo e, em particular, a 
possibilidade de minimizar os erros de confusão, escolhendo-se um número de classes adequado e definindo-se amostras, em conjunto com os parâmetros dos algoritmos, para diminuir a confusão; de outro lado, esses fatores, que contribuem para melhorar o desempenho dos classificadores, requerem treinamento mais elaborado da pessoa ou pessoas executando o trabalho de classificação e exigem um trabalho mais demorado e primoroso de análise dos dados prévia e posteriormente à execução do algoritmo, de modo que possa haver casos em que o mesmo resultado final possa ser obtido pela correção dos resultados do classificador automático com o auxílio de ferramentas de edição oferecidas nos sistemas de processamento de imagens e SIGs. Tais ferramentas podem, em certos casos, oferecer possibilidades de treinar a pessoa ou equipe a custos menores, em comparação com o treinamento mais sofisticado em técnicas de classificação digital. Finalmente, é preciso também considerar os requisitos de qualidade condicionados pela aplicação final do mapa a ser produzido: um aspecto particularmente importante nos estudos de mudanças de cobertura e uso da terra é garantir que o erro de classificação seja significativamente inferior às taxas de mudança, o que pode colocar requisitos adicionais mais severos sobre o trabalho, cuja satisfação não é necessariamente garantida pela escolha de um algoritmo específico, mas pela concepção do trabalho como um todo.

\section{CONCLUSÕES}

Os classificadores K-médias e MaxVer apresentaram resultados satisfatórios, sendo o método supervisionado (MaxVer) mais adequado na área "Espinha de peixe". Há pelo menos dois fatores que devem ser considerados na escolha do método de classificação: $(a)$ a alta variabilidade espectral de uma mesma classe de uso e também entre as cenas, devido a variações no sensor entre as passagens do satélite; e $(b)$ a própria dinâmica espacial de ocupação do território, que influencia nas classes de uso, nos processos de transição entre essas classes e nas bordas. Assim, um mapeamento satisfatório não constitui tarefa simples e não se dá pelo uso de uma única metodologia, mas, sim, requer análise prévia das classes de uso quanto à sua natureza e dinâmica, seguida preferencialmente por um método híbrido de mapeamento. É possível esperar diferentes desempenhos para os vários algoritmos de classificação em condições de diferentes configurações das áreas de estudo, sendo necessário considerar os requisitos da aplicação final do estudo e os custos de treinamento e operação de uma equipe. Trata-se, dessa forma, de um problema que poderia ser comparado a um problema de engenharia do estudo de cobertura e uso da terra, em que deve ser considerada a relação custo/benefício das diversas alternativas possíveis antes de ser escolhida uma solução entre várias; ou, nos casos em que considerações de custo não necessitem ser contabilizadas explicitamente, os comentários citados anteriormente podem inspirar um trabalho orientado para um esforço mais sistemático de avaliação prévia de várias opções sob os parâmetros de cada projeto, antes de se iniciar a produção final de um mapa de cobertura e uso da terra.

\section{AGRADECIMENTOS}

Aos projetos FAPESP(95/2432-5), CNPq (301474/91) e FINEP (0816/95, Contrato 6.6.96.0474.00), pelo apoio.

\section{REFERÊNCIAS}

ALVES, D. S. et al. Characterizing landscape changes in central Rondônia using Landsat TM imagery. International Journal of

Remote Sensing, v.20, n.14, p.2877-2882, 1999.

ALVES, D. S. Space-times dynamics of deforestation in Brazilian Amazon.

International Journal of Remote Sensing, v.23, n.14, p.2903-2908, 2002.

ALVES, D. S. et al.. Land use intensification and abandonment in Rondônia, Brazilian Amazonia. International Journal of Remote Sensing, v.24, n.4, p.899-903, 2003.

ALVES, D. S. Science and technology and sustainable development in Brazilian Amazon. In: TSCHARNTKE, T. et al. (Eds.), The stability of tropical rainforest margins. Berlin: Springer, 2007a. p.493-512.

ALVES, D. S. Cenários de cobertura e uso da terra e dimensões humanas no LBA. In: BECKER, B.; ALVES, D.; COSTA, W. (Orgs.). Dimensões humanas da biosfera-atmosfera na Amazônia. São Paulo: EDUSP, 2007b. p.39-63.

ALVES, D. S. et al. The changing rates and patterns of deforestation and land use in Brazilian Amazonia. In: KELLER, M. et al. (Eds.)

Amazonia and global change. Washington, DC: AGU, 2009. p.11-23. (Geophysics Monograph Series, 186).

Revista Árvore, Viçosa-MG, v.36, n.3, p.537-547, 2012 
ARAÚJO, R.; LENÁ, P. Da predação à sustentabilidade na Amazônia: a difícil metamorfose. In: ARAÚJO, R.; LENÁ, P. (Eds.). Desenvolvimento sustentável e sociedades na Amazônia. Belém: Museu Goeldi, 2010. p.13-53.

ASNER, G. P. et al. Selective logging in the Brazilian Amazon. Science, v.310, p.480-482, 2005.

ASNER, G. P. From the cover: condition and fate of logged forests in the Brazilian Amazon. Proceedings of the National Academy of Sciences - PNAS, v.103, n.34, p.1294712950, 2006. Disponível em: <http://www.pnas.org/ content/103/34/12947>full

BATISTELLA, M.; ROBESON, S.; MORAN, E. F. Settlement design, forest fragmentation, and landscape change in Rondonia, Amazonia, Photogrammetric Engineering and Remote Sensing, v.69, p.805-812, 2003.

BATISTELLA, M.; MORAN, E. F. Dimensões humanas do uso e cobertura das terras na Amazônia: uma contribuição do LBA, Acta Amazonica, v.35, n.2, p.239-247, 2005.

BISHOP, Y. M.; FEINBERG, S. E.; HOLLAND, P. W Discrete multivariate analysis: theory and practice. Cambridge: Mit Press, 1975. 357p.

CONGALTON, R. G.; MEAD, R. A. A quantitative method to test for consistency and correctness in photointerpretation. Photogrammetric Engineering and Remote Sensing, v.49, n.1, p.69-74, 1983.

COSTA, F. A. Heterogeneidade estrutural e trajetórias tecnológicas na produção rural da Amazônia. In: BATISTELLA, M. et al. (Eds.) Amazônia: natureza e sociedade e transformação. São Paulo: EDUSP, 2008. p.137-180.

FAMINOW, M. D. Cattle deforestation and development in the Amazon. New York: CAB International, 1998.

FIDALGO, E. C. C. et al. Mapeamento do uso e da cobertura atual da terra para indicação de áreas disponíveis para reservas legais: estudo em nove municípios da região Amazônica. Revista Árvore, v.27, n.6, p.871-877, 2003.
FOODY, G. M. et al. Mapping tropical forest fractional cover from coarse spatial resolution remote sensing imagery. Plant Ecology, v.131, n.2, p.143-154, 1997.

HECHT, S. B.; NOORGAARD, R. B.; POSSIO, G. The economics of cattle ranching in Eastern Amazonia. Interciencia, v.13, p.233-240, 1988.

INSTITUTO NACIONAL DE COLONIZAÇÃO E REFORMA AGRÁRIA - INCRA. Dossiê - Divisão de Assentamento. Porto Velho: 1996. (Relatório Interno)

LAMBIN, E. F.; GEIST, H. J.; LEPERS, E. Dynamics of land-use and land-cover change in tropical regions. Annual Review

Environment Resources, v.28, p.205-241, 2003.

LANDIS, J. R.; KOCH, C. H. The measurement of observer agreement for categorical data. Biometrics, v.33, n.3, p.159-174, 1977.

LINHARES, C. A.; SOARES, J. V.; BATISTA, G. T. Influência do desflorestamento na dinâmica da resposta hidrológica na Bacia do Rio Ji-Paraná/ RO. In: SIMPÓSIO BRASILEIRO DE SENSORIAMENTO REMOTO, 12., Goiânia, 2005. Anais... São José dos Campos: INPE, 2005. p.3097-3105.

LINHARES, C. A. et al. Deforestation and hydrology dynamic in Ji-Paraná river basin, Brazil. In: SIMPÓSIO BRASILEIRO DE

SENSORIAMENTO REMOTO, 13.,2007, Florianópolis. Anais... Florianópolis: 2007. p.6799-6806.

LU, D. et al. Classification of successional forest stages in the Brazilian Amazon basin. Forest Ecology and Management, v.181, p.301312,2003 .

LU, D. et al. Change detection techniques. International Journal of Remote Sensing, v.25, n.12, p.2365-2407, 2004.

KAIMOWITZ, D. et al. Hamburger connection fuels amazon destruction. Bangor: Center for International Forest Research, 2004. Disponível em: http://www.cifor.cgiar.org/ publications/pdf_files/media/Amazon.pdf 
MACHADO, L. A fronteira agrícola na Amazônia. In: BECKER, B. K. et al. (Ed.) Geografia e meio ambiente no Brasil. 1998. p.181-217.

MARGULIS, S. Causas do desmatamento da Amazônia Brasileira. Brasília: Banco Mundial, 2003. 100p.

MELLO, A. Y. I.; ALVES, D. S. Secondary vegetation dynamics in the Brazilian Amazon based on thematic mapper imagery. Remote Sensing Letters, v.2, n.3, p.189-194, 2011.

MERTENS, B. et al. Crossing spatial analyses and livestock economics to understand deforestation processes in the Brazilian Amazon: the case of São Félix do Xingu in South Pará. Agricultural Economics, n.27, p.269-294, 2002.

MORAN, E, F. et al. Integrating Amazonian vegetation, land-use, and satellite data. BioScience, v.44, n.5, p.329-338, 1994.

MORAN, E. F.; OSTROM, E. (Orgs.). Ecossistemas florestais: interação homemambiente. São Paulo: SENAC/EDUSP, 2009. 544p

NEPSTAD, D. C. et al. Large-scale impoverishment of Amazonian forests by logging and fire, Nature, v.398, p.505-508, 1999.

NEPSTAD, D. et al. Road paving, fire regime feedbacks, and the future of amazon forests". Forest Ecology and Management, v.5524, p.1-13, 2001.

PEREIRA, M. C.; SETZER, A. W. Spectral characteristics of fire scars in Landsat-5 TM images of Amazonia. International Journal of Remote Sensing, v.14, n.11, p.2061-2078, 1993.

PONZONI, F. J.; REZENDE, A. C. P. Caracterização espectral de estágios sucessionais de vegetação arbórea secundária arbórea em Altamira (PA), através de dados orbitais. Revista Árvore, v.28, n.4, p.535-545, 2004.

ROBIN, A.; MOISAN, L.; LE HEGARATMASCLET, S. An unsupervised approach for subpixelic land-cover change detection. In: INTERNATIONAL WORKSHOP ON THE ANALYSIS OF MULTITEMPORAL REMOTE SENSING IMAGES, 4., 2007. Proceedings... 2007. p.1-6.
RUDEL, T. K. et al. Forest transitions: towards a global understanding of land use change.

Global Environmental Change, v.15, n.1, p.23-31, 2005.

SANO, E. E. et al. Levantamento do uso atual da terra através de imagens do Landsat 5-TM na microregião de Tomé-Açu e alguns municípios das microregiões do Baixo Tocantins e Guajarina (PA). Belém: SUDAM-CHSRA/ OEA, 1989.67p.

SCHMINK, M.; WOOD, C. H. Contested frontiers in Amazonia. New York: Columbia University Press, 1992.

SHIMABUKURO, Y. E.; SANTOS, J. R.; AQUINO, L. C. S. Vegetation survey in Amazonia using Landsat data. São José dos Campos: INPE, 1982.7p. (INPE-2409-PRE/118)

SILVA, G. B. S.; BETIOL, G. M.; SANO, E. E. Análise comparativa de quatro métodos de classificação supervisionada para a discriminação de fitofisionomias de Cerrado. Geografia. Ensino \& Pesquisa - UFSM, v.12, p.39353947, 2008.

SKOLE, D.; TUCKER, C. Tropical deforestation and habitat fragmentation in the amazon - satellite data from 1978 to 1988 . Science, v.260, p.19051910, 1993.

SOARES-FILHO, B. S. et al. Cenários de desmatamento para a Amazônia. Estudos Avançados, v.19, n.54, p.137-152, 2005.

STEININGER, M. K. Satellite estimation of tropical secondary forest above-ground biomass : data from Brazil and Bolivia. International Journal of Remote Sensing, v.21, n.6-7, p.1139-1157, 2000.

TARDIN, A. T. et al. Subprojeto desmatamento: convênio IBDF/CNPq - INPE. São José dos Campos: INPE, 1980. (Technical Report, INPE-1649-RPE/103)

VERBURG, P. H.; NEUMANN, K.; NOL, L. Challenges in using land use and land cover data for global change studies. Global Change Biology, v.17, n.2, p.974-989, 2011.

Revista Árvore, Viçosa-MG, v.36, n.3, p.537-547, 2012 